\section{Healthy ageing: From a myth to a reality}

The dream of living long and healthy has accompanied man since the beginning of time. The present remarkable increased life expectancy is most probably the result of better living conditions, medical progress, and better health care organization. Dramatic demographic changes occur not only in industrialised countries, but primarily affect the least developed part of the world. This extraordinary success of humanity brings with it many challenges to build a future with healthy seniors. The recently released World report on ageing and health [1], one of the most important WHO documents in recent years, confirms the crucial importance of the maintenance of functional ability throughout life. The myth that old age is always associated with physical or economic dependence is refuted $[1,2]$. Michel et al. thoughtfully analyse the possible interventions to promote functionally independent ageing [3]. "Distal" risk factors (economic and sociocultural determinants, education, air pollution, etc.) are distinguished from "intermediate" and "proximal" factors. Interventions contrasting "intermediate" risk factors include actions on health habits and behaviours. Interventions against "proximal" risk factors include actions on risk factors related to diseases that increase disability; i.e., vaccination programs [4], prevention of dementia, cardiovascular disease, diabetes, COPD, musculoskeletal diseases, sensory deficiencies, inappropriate drug prescriptions, and strategies to delay, prevent or reverse the frailty process [3].

The traditional WHO definition of health as "complete physical, mental and social well-being" includes components beyond the mere absence of disease. R. Smith, former editor of BMJ, pointed out that "it would leave most of us unhealthy most of the time" [5], since this is unreachable for most people in the world, and may contribute to medicalization of the society. In 2008, Jadad and O'Grady in a BMJ editorial called for a "global conversation" through a blog, on how to define health [6]. Later on, along with a group of international contributors, a new definition of health as "the ability to adapt and to self-manage in response to physical, mental, or social challenges" was proposed [7]. Facing an ageing global population with an increasing burden of chronic diseases, this definition emphasizes the role of the human capacity to cope autonomously the changing physical, emotional and social problems during ageing, and highlights the possibility to keep participating and functioning with a feeling of well-being, even in the presence of chronic diseases, as described in a global collaboration, in which we participated [8]. In our everyday clinical practice, we witness examples of this human ability, called "resilience" [9].

Active and healthy ageing is not only a societal "duty". It is also a personal responsibility. Everyone eventually has the old age that deserves. This makes us think on the opportunity, at any age, to care for the own ageing. Lifestyle and behaviour across the life course (physical exercise, adequate nutrition, non-smoking) are fundamental. It is for this purpose that we all need to build and disseminate a culture of "Active and healthy ageing". In parallel, preventing diseases that increase disability, and delaying or preventing frailty and dependency [10], need to become a core focus of public health actions.

\section{Disclosure of interest}

The authors declare that they have no competing interest.

\section{References}

[1] WHO. World report on ageing and health; 2015 [Geneva]http://www.who.int/ ageing/events/world-report-2015-launch/en/.

[2] Bousquet J, Kuh D, Bewick M, Strandberg T, Farrell J, Pengelly R, et al. Operative definition of active and healthy ageing (AHA): meeting report, Montpellier October 20-21, 2014. Eur Geriatr Med 2015;6:196-200. http://dx.doi.org/ 10.1016/j.eurger.2014.12.006.

[3] Michel JP, Dreux C, Vacheron A. Healthy ageing: evidence that improvement is possible at every age. Eur Geriatr Med 2016 [in press].

[4] McElhaney JE, Gavazzi G, Flamaing J, Petermans J. The role of vaccination in successful independent ageing. Eur Geriatr Med 2016;7:171-5. http:// dx.doi.org/10.1016/j.eurger.2016.01.007.

[5] Smith R. BMJ 2008, http://blogs.bmj.com/bmj/2008/07/08/richard-smiththe-end-of-disease-and-the-beginning-of-health/.

[6] Jadad AR, O'Grady L. How should health be defined? BMJ 2008;337:a2900. http://dx.doi.org/10.1136/bmj.a2900.

[7] Huber M, Knottnerus JA, Green L, van der Horst H, Jadad AR, Kromhout D, et al. How should we define health? BMJ 2011;343:d4163. http://dx.doi.org/ 10.1136/bmj.d4163.

[8] Jadad AR, Cabrera A, Lyons RF, Martos F, Smith R, et al. When people live with multiple chronic diseases: a collaborative approach to an emerging global challenge. Granada: Andalusian School of Public Health; 2010, http://www. opimec.org/media/files/BOOK_OPIMEC_100818.pdf.

[9] Rockwood K, Mitnitski A. Resilience and frailty: Further steps, best taken together. Eur Geriatr Med 2015;6:405-7. http://dx.doi.org/10.1016/j.eurger.2015.06.001.

[10] Strandberg TE, Pitkälä KH, Tilvis RS. Frailty in older people. Eur Geriatr Med 2011;2:344-55. http://dx.doi.org/10.1016/j.eurger.2011.08.003.

M. Barbagallo*, L.J. Dominguez

Geriatric unit, department of internal medicine and geriatrics, university of Palermo, Viale F. Scaduto 6/c, 90144 Palermo, Italy

*Corresponding author. Tel.: +39 0916552885; fax: +39 0916552952

E-mail address: mario.barbagallo@unipa.it (M. Barbagallo)

Received 26 April 2016

Accepted 27 April 2016

Available online 1 July 2016 